\title{
Qualitätsmessung mit Routinedaten im Pflegeheim am Beispiel Dekubitus
}

\section{Pressure ulcer in German Nursing Homes: Quality Assessment Using Claims Data of Statutory Health and Long-Term Care Insurance}

\section{(ㄷ)(1) (우 $\Theta$}

\author{
Autoren \\ Susann Behrendt ${ }^{1}$, Antje Schwinger ${ }^{1}$, Chrysanthi Tsiasioti ${ }^{1}$, Kai Stieglitz $^{1}$, Jürgen Klauber ${ }^{2}$
}

Institute

1 Wissenschaftliches Institut der AOK (WIdO), Fachbereich Pflege, Berlin

2 Wissenschaftliches Institut der AOK (WIdO), Geschäftsführung, Berlin

Key words

Quality, indicator, long-term care, claims data, pressure ulcer

\section{Bibliografie}

DOI https://doi.org/10.1055/a-1057-8799

Online-Publikation: 4.2.2020

Gesundheitswesen 2020; 82 (Suppl. 1): S52-S61

(c) Georg Thieme Verlag KG Stuttgart · New York

ISSN 0949-7013

Korrespondenzadresse

Susann Behrendt

WIdO, Pflege, Rosenthaler Straße 31

10178 Berlin

susann.behrendt@wido.bv.aok.de

\section{ZUSAMMENFASSUNC}

Ziel Obwohl Routinedaten des Gesundheitswesens Teil der gesetzlichen Qualitätssicherung in der SGB-V-Versorgung sind, finden sie keinen Eingang in die Qualitätsmessung in der Langzeitpflege (SGB XI). Routinedaten der GKV und SPV liefern jedoch wertvolle Informationen zur Ergebnisqualität der Versorgung in Pflegeheimen. Die routinedatenbasierte Qualitätsmessung im Pflegeheim ist kaum erforscht und entsprechende Basisarbeit in der Sekundärdatennutzung geboten. Dies betrifft neben dem Datenlinkage ebenso neue Operationalisierungswege für pflegenahe Qualitätsindikatoren und deren Risikoadjustierung für faire Heimvergleiche. Am Beispiel des Dekubitus-Auftretens im Pflegeheim entwickelt der Beitrag einen routinedatenbasierten Qualitätsindikator und diskutiert Potenziale und methodische Herausforderungen.

Material und Methoden Die Analyse basiert auf Daten aller elf AOK Kranken- und Pflegekassen (2015) und umfasst 31\% der
Pflegeheime in Deutschland. Die Operationalisierung des im Heim erworbenen Dekubitus bezog ICD-10-Diagnosen und Arzneimitteldaten zu Verbandsstoffen ein. Die Eignung von Abrechnungsdaten zu dekubitusspezifischen Hilfsmitteln wurde in diesem Zusammenhang ebenso getestet. Die Risikoadjustierung orientierte sich an der Qualitätssicherung mit Routinedaten im Krankenhaus (QSR). Der Berechnung der Standardized Morbidity Ratio, der Relation von beobachteter und angesichts der Risikostruktur des Heimes erwarteter Dekubitus-Rate je Heim, lag eine logistische Regression mit robusten Standardfehlern zugrunde.

Ergebnis und Schlussfolgerungen 2015 trat bei 7,2\% der Bewohner mindestens ein Dekubitus im Pflegeheim neu auf. Es zeigten sich deutliche Qualitätsunterschiede zwischen den Einrichtungen. In der Gesamtschau ist die routinedatenbasierte Messung des Dekubitus-Auftretens im Pflegeheim machbar und kann in Form von Ergebnisqualitätsindikatoren zu Versorgungstransparenz und -evaluationen im Pflegeheim beitragen. Informationen im Rahmen der Einschätzung der Pflegebedürftigkeit sowie der novellierten gesetzlichen Qualitätsmessung eröffnen routinedatenbasierten Qualitätsindikatoren in der Langzeitpflege künftig neue Wege der Operationalisierung von pflegenahen Aspekten.

\section{ABSTRACT}

Objective Although administrative data on health care in Germany are part of legal quality assurance in hospital care, they are not part of quality assessment in long-term care. However, claims data of German statutory health and long-term care insurance provide valuable information on outcome quality in nursing homes. Claims data-based quality measurement in nursing homes has hardly been researched and basic work in secondary data analysis is required. This involves the claims data linkage of both statutory health and long-term care insurance as well as new ways of operationalization for quality indicators and their risk adjustment for fair facility comparisons. Using the example of pressure ulcer (PU) occurrence in nursing homes, this study develops a claims data-based quality indicator and discusses potentials and methodological challenges. 
Methods The analysis is based on administrative data from eleven statutory health and long-term care insurance funds (AOK, 2015). The dataset covers $31 \%$ of German nursing homes. The operationalisation of PU acquired within the facility included ICD-10 diagnoses, and prescriptions on dressings. Relevance and validity of claims data on PU-specific aids were also checked in this context. Our risk adjustment strategy followed the one already established by the claims data-based QSR (Quality assurance of inpatient health-care). The Standardized Morbidity Ratio was based on logistic regression with robust standard errors.
Results and conclusion In 2015, $7.2 \%$ of the nursing home residents had at least one PU incident within the facility. The outcome quality considerably varied between facilities. Overall, claims data-based measurement of PU occurrence as outcome quality indicator is feasible for inpatient long-term care and can contribute to transparency and evaluation of care in nursing homes. Information derived from an assessment of care dependency as well as within the amended legal quality assurance system for long-term care may offer new opportunities for routine data-based quality indicators in nursing homes.

\section{Einleitung}

Die Nutzung von Routinedaten der gesetzlichen Krankenversicherung steht seit Jahren auf der Agenda der externen gesetzlichen Qualitätssicherung im Krankenhaus. In der Pflegeversicherung, die nach 10 Jahren Pflegenoten-Kritik auf dem Prüfstand ist, zeigt sich hingegen ein ganz anderes Bild: Hier ist der Einsatz von Routinedaten auch im Rahmen der aktuellen Novellierung weiterhin nicht vorgesehen. Zwar wird es perspektivisch neben den Qualitätsprüfungen vor Ort Indikatoren zur Messung der Ergebnisqualität im Pflegeheim geben. Diese basieren jedoch auf Eigendokumentationen der Einrichtungen und betrachten lediglich pflegenahe (SGB XI-) Versorgungsaspekte [1]. Medizinische und sektorenübergreifende Bereiche werden ausgeblendet - und damit ein Stück Versorgungsrealität für die multimorbiden, betagten Bewohner (siehe auch [2, 3]). Demgegenüber bieten Routinedaten der Sozialversicherungsträger die Chance, die Qualitätsmessung in der stationären Langzeitpflege zu stärken, da sich auf ihrer Basis die pflegerische und medizinische Versorgung erfassen lässt. Für das Setting Pflegeeinrichtung, in dem ärztliche, therapeutische und pflegerische Akteure und Prozesse ineinandergreifen, ist dies von erheblicher Bedeutung. 2012 forderte auch der Sachverständigenrat für die Entwicklung im Gesundheitswesen die Nutzung von Routinedaten für die Qualitätssicherung in der Pflege [4].

Das routinedatengestützte Qualitätsassessment und -reporting für die vollstationäre Langzeitpflege ist dennoch von einer Etablierung weit entfernt. International sind die USA mit dem Nursing Home Compare-System zwar Vorreiter beim Qualitätsreporting über Pflegeheime; jedoch werden auch hier erst seit 2017 Sekundärdaten, d. h. personenbezogene Medicare-Abrechnungsdaten für den Outcome-Qualitätsindikator zu Hospitalisierungen von Pflegeheimbewohnern (Kurzzeitpflege) einbezogen [5]. In Deutschland existiert Forschung in diesem Bereich quasi nicht. Eine Ausnahme stellt die Studie von Przylog et al. (2016) zum Zusammenhang von Verletzungen und Pflegenoten der Einrichtungen unter Verwendung von Abrechnungsdaten einer gesetzlichen Krankenkasse dar [6]. Gleichwohl ist die Qualitätsmessung mittels Abrechnungsdaten der medizinischen Leistungserbringung in Deutschland und international seit vielen Jahren Forschungsfeld und Praxis (u.a. [7-11]).

Vor diesem Hintergrund entwickelt der Beitrag am Beispiel Dekubitus eine routinedatenbasierte, indikatorgestützte Messung von Ergebnisqualität im Pflegeheim und testet diese empirisch. Be- tagte Pflegebedürftige sind durch ihr Alter, ein geschwächtes Immunsystem und ihre Multimorbidität als besonders gefährdete Risikogruppe für Dekubitus einzustufen. Dennoch ist die Erkrankung keine „schicksalhafte Begleiterscheinung des Alters“ [12], sondern gilt nach wissenschaftlichen Erkenntnissen durch Präventionsmaßnahmen als in der Regel vermeidbar [13, 14]. In Ausnahmefällen, in denen ein Dekubitus nicht vermeidbar ist, geht dies zumeist auf eine veränderte Prioritätensetzung in der Versorgung bspw. von palliativ versorgten, bettlägerigen Menschen zurück [15, 16]. Der Beitrag schließt mit den methodischen Herausforderungen des Verfahrens sowie den Perspektiven, die sich künftig durch die novellierten Prüfinstrumentarien ergeben.

\section{Methodik}

\section{Daten und Stichprobe}

Grundlage der Indikatorentwicklung zum Dekubitus-Auftreten im Pflegeheim ist eine Analyse von anonymisierten AOK-Abrechnungsdaten der Kranken- und Pflegekassen der Jahre 2014 und 2015 ( Abb. 1).

\section{Operationalisierung des Indikators zum Dekubitus- Auftreten im Pflegeheim}

Eine Operationalisierung des Auftretens eines Dekubitus im Pflegeheim ausschließlich mittels ärztlich dokumentierter Diagnosen wurde als nicht zielführend erachtet. Rund ein Zehntel $(9,2 \%)$ der Pflegeheimbewohner im Durchschnitt der Quartale ( $n=172531$; Bewohneranzahl im Durchschnitt der Quartale) wiesen hier mindestens eine als gesichert dokumentierte Vertragsarztdiagnose Dekubitus (ICD-10-GM: L89* ) auf. Ein systematischer Review zur Epidemiologie von Dekubitus in Deutschland kommt demgegenüber auf Basis von 25 Primärerhebungen auf einen Prävalenz-Range von 2,7 \% bis 6,7 \% und auf eine Inzidenz in Höhe von 3,1 \% [17]. Dem MDS-Bericht zur Qualität in der stationären Pflege (2018) zufolge erkrankten 3,9\% der Bewohner im Jahr 2016 an Dekubitus ([18]).

Die Sekundärdatenforschung im Gesundheitswesen belegt hinreichend, wie wichtig eine interne Validierung ist, um die Abrechnungsdiagnose zu bestätigen [19-21]. Eine valide Identifizierung der an Dekubitus im Pflegeheim erkrankten Bewohner als Outcomevariable ist Voraussetzung für eine belastbare Qualitätsmes- 


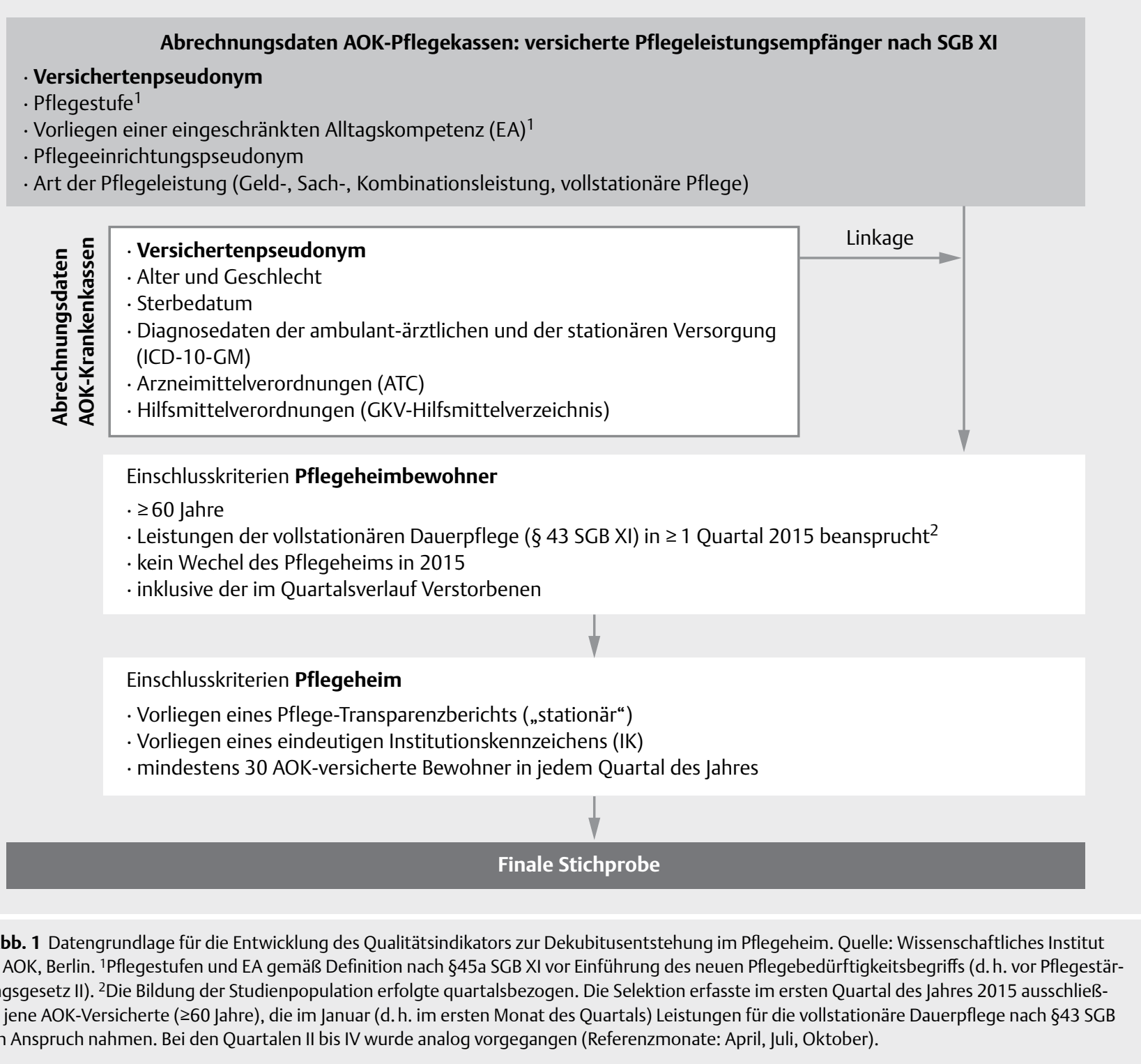

sung. Die vorliegende Analyse betrachtete aus diesem Grund neben der vertragsärztlichen Diagnose Dekubitus, inwieweit der zusätzliche Einbezug von dekubitusspezifischen Leistungsziffern, konkret die Verordnungen von Verbandsmaterial im Rahmen der Wundversorgung ( Tab. 1) sowie dekubitusspezifische Hilfsmittel gemäß Hilfsmittelverzeichnis der gesetzlichen Krankenversicherung (GKV) $[14,22]$ eine belastbare Schätzung der Erkrankungshäufigkeit unterstützen kann. Inhaltlich ausschlaggebend für die Auswahl der Verbandsmaterialien war die internationale Leitlinie zur Prävention und Behandlung von Dekubitus des National sowie des European Pressure Ulcer Advisory Panel zusammen mit der Pan Pacific Pressure Injury Alliance (NPUAP/EPUAP/PPPIA). Hinzugezogen wurden zudem die Informationen des Expertenstandards zur Dekubitusprophlyaxe des Deutschen Netzwerks für Qualitätsentwicklung in der Pflege ([13]).

Bei Dekubitus Kategorie II ist leitlinienkonform zwingend ein Wundverband erforderlich. Maßnahmen der Druckentlastung sind bei Bedarf durch druckverteilende Hilfsmittel zu ergänzen. Während eine vertragsärztliche gesicherte Dekubitusdiagnose und eine entsprechende Hilfsmittelverordnung innerhalb eines Quartals bei lediglich 1,4\% der Stichprobe auftrat, führte der Algorithmus aus vertragsärztlicher gesicherter Diagnose und Verbandsverordnungen innerhalb desselben Quartals zur plausiblen Dekubitus-Prävalenz von $4 \%$. Dies trifft den Prävalenzbereich der oben referierten Studie und des MDS-Berichts [17, 18]. \ Abb. 2 zeigt den finalen Indikator „Anteil der Bewohner mit neu aufgetretenem Dekubitus je Pflegeheim“ (kurz: Dekubitus-Indikator). Um lediglich jene Ereignisse zu erfassen, die in den Pflegeeinrichtungen neu auftreten, schärfte ein Inzidenzkriterium die routinedatenbasierte Definition von Dekubitus im Pflegeheim.

\section{Risikoadjustierung}

Faire indikatorgestützte Qualitätsvergleiche zwischen Pflegeeinrichtungen bedürfen der Berücksichtigung der über die Pflegehei- 
me unterschiedlich verteilten Risiken für das Indikatorereignis in der Bewohnerschaft. Dabei geht es um die nicht von der Einrichtung selbst beeinflussbaren Faktoren ([23-25]). Ein weit verbreitetes Verfahren der Risikoadjustierung für Qualitätsindikatoren ist

- Tab. 1 Verbandsmaterial für die Wundversorgung bei Dekubitus - einbezogene Produktgruppen nach PZN-Klassifikation zur Identifizierung der Dekubitusfälle auf Routinedatenbasis. Quelle: Wissenschaftliches Institut der AOK, Berlin.

\begin{tabular}{|l|}
\hline PZN-Klassifikation: Gruppe \\
\hline Schaumverbände feinporig \\
\hline Superabsorber/Vlieskompresse mit Superabsorber \\
\hline Hydrokolloide normal \\
\hline Alginate \\
\hline Folien/semipermeable Transparentfolien \\
\hline Hydrogel \\
\hline Hydrofaser/-fiber/Aquafaser \\
\hline Aktive Wundauflagen \\
\hline Aktivkohleverbände \\
\hline Hydrokolloidähnliche Wundauflagen \\
\hline Wundspülungen konserviert \\
\hline Honig-Produkte \\
\hline Hydrophobe Wundauflagen \\
\hline Saugspülkörper zur Nasstherapie \\
\hline Sonstige Wundauflagen \\
\hline Produkte zur Unterstützung der Wundreinigung \\
\hline Moderne Post-OP-Verbände \\
\hline Wundauflagen aus feuchter Zellulose/Sonstige Wundauflagen \\
\hline Hautschutzpräparate \\
\hline Produkte für Spalthautentnahmestellen \\
\hline
\end{tabular}

die multivariate logistische Regression ([5, 23, 26-29]). Mit ihr lassen sich multiple Risikofaktoren flexibel in ein Modell einbeziehen und so der multifaktoriellen Bedingung von Ereignissen, hier der Dekubitusgenese, Rechnung tragen.

Die Auswahl dekubitusrelevanter Risikofaktoren erfolgte leitliniengestützt [14]. Die Herausforderung bestand hierbei in der validen Übersetzung der in > Tab. 2 gelisteten Einflussfaktoren auf Routinedatenbasis. Der Pflegeheimbewohner war von der jeweiligen Erkrankungsgruppe (Einflussfaktor) betroffen, sofern er eine ambulantärztliche gesicherte Diagnose aus der Erkrankungsgruppe in zwei unterschiedlichen Quartalen (M2Q) oder mindestens eine stationäre Haupt-/Nebendiagnose (Entlassung; M1K) im Jahr 2015 aufwies.

Die Operationalisierung der Risikofaktoren Inkontinenz, Diabetes mellitus, Malnutration und eingeschränkte Mobilität orientierte sich an jenen Diagnosesets, welche das IQTIG im Rahmen des gesetzlichen QS-Verfahrens Dekubitusprophylaxe bei der Risikoadjustierung der im Krankenhaus neu aufgetretenen Dekubiti einbezieht [30]. Zur besonderen Berücksichtigung der geriatrietypischen Multimorbidität wurden diese Listen um Diagnosecodes der sogenannten geriatrietypischen Merkmalskomplexe für Mobilitätseinschränkungen/Immobilität, Sensorik-Störungen, Inkontinenz sowie Malnutration ergänzt [31]. Für den Risikofaktor „allgemein schlechter Gesundheitszustand “ wurde die Pflegestufe sowie das Vorliegen einer eingeschränkten Alltagskompetenz nach §45a SGB XI (vor PSG II; in der Regel einer Demenz, kurz: EA) als Surrogat angesetzt. Beide Aspekte stehen für die Pflegebedürftigkeit des Bewohners und beschreiben das Ausmaß körperlicher, geistiger und psychischer Beeinträchtigungen sowie dem daraus resultierenden Unterstützungsbedarf. Faktoren wie Hautzustand und erhöhte Körpertemperatur sind nicht Bestandteil des ICD-10 bzw. der medizinischen Leistungsabrechnung und damit auf dieser Datengrundlage nicht abbildbar.

\section{routinedatenbasierter Indikator Dekubitus}

Zähler: Bewohner mit mindestens einem im Pflegeheim neu aufgetretenem Dekubitus

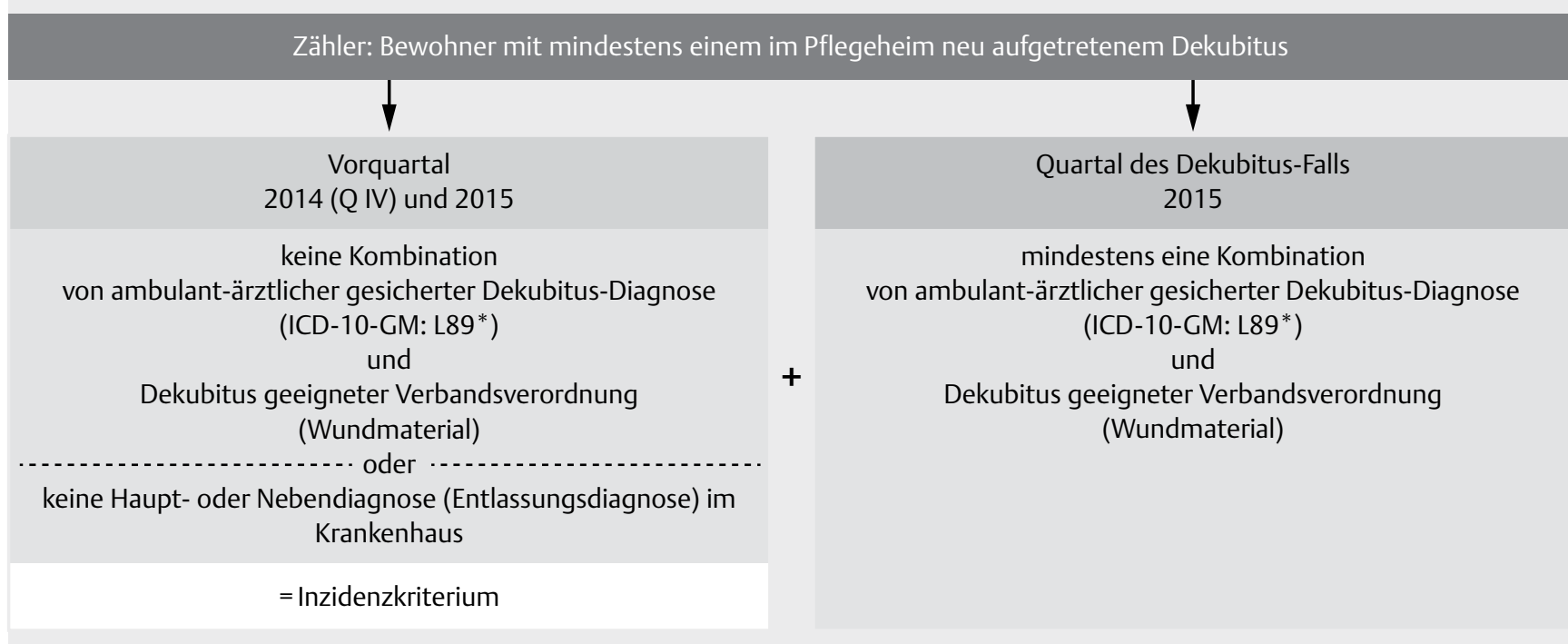

Nenner: Pflegeheimbewohner

Abb. 2 Operationalisierung des Indikators zum Dekubitus-Auftreten im Pflegeheim. Quelle: Wissenschaftliches Institut der AOK, Berlin. 
> Tab. 2 Einflussfaktoren auf die Dekubitusgenese für eine routinedatenbasierte Adjustierung des Indikators. Quelle: Wissenschaftliches Institut der AOK, Berlin nach NPUAP 2014 [14].

\begin{tabular}{|c|c|}
\hline Risikofaktoren Dekubitusgenese & $\rightarrow$ Merkmale auf Routinedatenbasis \\
\hline Alter & Geburtsjahr \\
\hline Geschlecht & $\mathrm{m}, \mathrm{w}$ \\
\hline Diabetes mellitus $^{1}$ & ICD-10-GM: E10, E11, E12, E13, E14 \\
\hline Mobilitätseinschränkungen ${ }^{1}$ & $\begin{array}{l}\text { ICD-10-GM: G81, G82, G83, M24, M24.5, M26, M62, M96.8, R26.3, R40, R46, S14, } \\
\text { S24, Z74, Z74.0, Z99, Z99.3 }\end{array}$ \\
\hline Aktivität & subsumiert unter Pflegestufe und Mobilitätseinschränkung \\
\hline Sensorik/Parkinson ${ }^{1}$ & ICD-10-GM: G20, R20, G50, G60, G61, G62, G63, G64 \\
\hline Malnutrition ${ }^{1}$ & ICD-10-GM: E41, E43, E44, E46, R63.3, R64 \\
\hline Dehydration $^{1}$ & ICD-10-GM: E86 \\
\hline Feuchtigkeit/Inkontinenz ${ }^{1}$ & ICD-10-GM: R32, R39.3, R39.4, R15 \\
\hline Hautzustand & nicht abbildbar \\
\hline Kategorie I Dekubitus & bereits vorliegende Dekubiti werden ausgeschlossen \\
\hline vorhandener Dekubitus & bereits vorliegende Dekubiti werden ausgeschlossen \\
\hline erhöhte Körpertemperatur & nicht abbildbar \\
\hline eingeschränkte Sauerstoffversorgung Haut/Durchblutung & nicht abbildbar \\
\hline $\begin{array}{l}\text { Blutwerte: erhöhtes C-reaktives Protein, Lymphopenie, } \\
\text { niedriges Albumin und Hämoglobin }\end{array}$ & nicht abbildbar \\
\hline ethnische Zugehörigkeit & nicht abbildbar \\
\hline allgemein schlechter Gesundheitszustand & $\begin{array}{l}\text { Pflegestufe I, II, III, III + Härtefalle und eingeschränkte Alltagskompetenz (Demenz) } \\
\text { als Surrogat für viele Aspekte wie Mobilität, Aktivität und allgemeiner Gesundheits- } \\
\text { zustand }\end{array}$ \\
\hline \multicolumn{2}{|c|}{$\begin{array}{l}\text { 'Der Pflegeheimbewohner war von der jeweiligen Erkrankungsgruppe (Risikofaktor) betroffen, sofern er eine ambulant-ärztliche gesicherte Diagnose } \\
\text { aus der Erkrankungsgruppe in zwei unterschiedlichen Quartalen (M2Q) oder mindestens eine stationäre Haupt-/Nebendiagnose (Entlassung; M1K) im } \\
\text { Jahr } 2015 \text { aufwies. Quelle: WIdO, nach: NPUAP } 2014 .\end{array}$} \\
\hline
\end{tabular}

Eine multiple logistische Regression (stepwise backwards) modellierte die Eintrittswahrscheinlichkeit der Zielvariable „mindestens ein neu aufgetretener Dekubitus im Pflegeheim in 2015“ (ja/nein) auf Bewohnerebene in Abhängigkeit der dekubitusspezifischen Risikostruktur. Die standardisierte Morbiditätsrate (SMR) setzte darauffolgend den beobachteten Anteil der Bewohner mit mindestens einem Dekubitus in Relation zum - angesichts der Risikostruktur erwarteten Wert. Jedes Pflegeheim erhielt so einen individuellen Score, der sich relational an einer über alle Pflegeheime vergleichbaren Risikostruktur orientierte. Dieses logistische Modell mit robusten Standardfehlern stellt eine Alternative zum logistischen Mehrebenenmodell dar. Beide Modelle geben korrekte Standardfehler wieder, jedoch beschreiben die geschätzten Koeffizienten inhaltlich verschiedene Aspekte [32]. Bei einem logistischen Mehrebenenmodell handelt es sich um konditionale Effekte, also innerhalb von Pflegeheimen (subjektspezifische Effekte), dagegen werden in einem logistischen Regressionsmodell population-averaged (durchschnittliche Effekte) bzw. marginale Wahrscheinlichkeiten berechnet, in die beide Varianzen innerhalb und zwischen Pflegeheimen mit in die Schätzung einfließen. Mehrebenenanalysen ermöglichen eine vollständige Betrachtung auf allen Analyseebenen, wo hingegen in einem Modell, das marginale Wahrscheinlichkeiten berechnet, ausschließlich die Regressionskoeffizienten von inhaltlichem Interesse sind. Dabei wird für die Interpretierbarkeit der Effekte die Abhängigkeitsstruktur methodisch korrigiert. Für die vorliegenden Analysen standen die marginalen Effekte im Fokus und aufgrund dessen wurde das konventionelle logistische Modell mit robusten Standardfehlern als methodisches Verfahren ausgewählt [32].

\section{Ergebnisse}

\section{Studienpopulation}

Die Studienpopulation umfasst 31,0\% ( $n=3459)$ der Pflegeheime mit vollstationärer Dauerpflege und 27,6\% ( $n=215863)$ aller Pflegeheimbewohner im Referenzjahr 2015 in Deutschland [33]. Knapp drei Viertel der betrachteten Pflegebedürftigen sind Frauen. Die Altersgruppe der über 80 -jährigen ist mit 75,4\% am stärksten besetzt. Rund zwei Drittel der Bewohner gelten als schwer- bzw. schwerstpflegebedürftig (Pflegestufe II bzw. III). Eine EA (primär Demenz) lag bei 69,3\% der Bewohner vor. $>$ Tab. 3 unterstreicht die Strukturähnlichkeit der Studienpopulation und der bundesweiten Pflegeheimbewohnerschaft gemäß Pflegestatistik. Deutliche Abweichungen finden sich bei der Trägerschaft und Heimgröße als Konsequenz der Einschlusskriterien (mindestens 30 AOK-Versicherte je Einrichtung - > deutlich weniger kleine und weniger private Pflegeheime).

Im Schnitt über alle Einrichtungen belegten AOK-Versicherte die Hälfte (49\%) der belegten Plätze je Einrichtung (einrichtungsbezogene Information der Pflegetransparenzberichte stationär, bezogen auf den Berichtstag). \ Abb. $\mathbf{3}$ zeigt jedoch auch, dass dieser Anteil zwischen den Pflegeheimen variiert.

\section{Indikator zum Dekubitus-Auftreten im Pflegeheim}

Gemäß Indikatordefinition traten im Jahr 2015 bei 7,2 \% der Bewohner (Studienpopulation, $\mathrm{n}=215863$ ) mindestens ein Dekubitus im Pflegeheim neu auf. Dies ist deutlich höher als die bei Operationalisierung berechnete Häufigkeit von 4,0\% auf Basis der Bewohner- 
- Tab. 3 Merkmale der Studienpopulation. Quelle: AOK-Daten 2015, Pflegestatistik 2015 (Statistisches Bundesamt 2017).

\begin{tabular}{|c|c|c|}
\hline & Stichprobe & $\begin{array}{l}\text { Pflegestatistik } \\
2015\end{array}$ \\
\hline \multicolumn{3}{|c|}{ Pflegeheimbewohner im Durchschnitt der Quartale 2015 in \% } \\
\hline Frauen & 74,8 & 73,3 \\
\hline \multicolumn{3}{|l|}{ Alter in Jahren } \\
\hline $60-70$ & 6,2 & 6,9 \\
\hline $70-80$ & 18,2 & 20,5 \\
\hline $80+$ & 75,6 & 72,6 \\
\hline \multicolumn{3}{|l|}{ Pflegestufe $^{1}$} \\
\hline 0 & 0,8 & 2,0 \\
\hline I & 37,0 & 38,1 \\
\hline II & 41,7 & 39,8 \\
\hline III + Härtefälle & 20,5 & 20,1 \\
\hline $\begin{array}{l}\text { eingeschränkte Alltagskompetenz nach } \S \\
45 \text { a SGB XI in \%1 }\end{array}$ & 69,3 & 73,0 \\
\hline \multicolumn{3}{|l|}{ Pflegeheimbewohner im Jahr 2015 in \% } \\
\hline verstorben & 23,4 & - \\
\hline \multicolumn{3}{|l|}{ Verweildauer im Pflegeheim in Monaten } \\
\hline mittlere Verweildauer & 9,6 & - \\
\hline 25. Perzentil & 9,2 & - \\
\hline 75. Perzentil & 10,1 & - \\
\hline \multicolumn{3}{|l|}{ Pflegeheime im ersten Quartal 2015 in \% } \\
\hline \multicolumn{3}{|l|}{ Träger } \\
\hline öffentlich & 5,1 & 4,8 \\
\hline freigemeinnützig & 61,5 & 53,0 \\
\hline privat & 32,8 & 42,2 \\
\hline \multicolumn{3}{|l|}{ Größe } \\
\hline 31-50 Plätze & 3,4 & 27,9 \\
\hline 51-150 Plätze & 86,4 & 66,1 \\
\hline 151 + Plätze & 10,3 & 6,0 \\
\hline \multicolumn{3}{|c|}{$\begin{array}{l}\text { 1Pflegestufen und EA gemäß Definition vor Einführung des neuen } \\
\text { Pflegebedürftigkeitsbegriffs im PSG II }\end{array}$} \\
\hline
\end{tabular}

zahl im Durchschnitt der Quartale $(n=172531)$ und damit auch der zeitpunktbezogenen Prävalenz im aktuellen MDS-Bericht. - Abb. 4 zeigt die erhebliche Variation des Indikatorwerts im Einrichtungsvergleich. Neben Pflegeheimen ohne derartige Dekubitusfälle waren bei $25 \%$ der Einrichtungen zwischen 9,8 und 27,5\% der Bewohnerschaft betroffen (4. Quartil). Damit erkrankten im auffälligen Quartil mehr als doppelt so viele Bewohner an Dekubitus wie im unauffälligen ersten Quartil.

Im Rahmen der Adjustierung dieser Ergebnisse mittels multipler logistischer Regression mit robust geschätzten Standardfehlern verblieben final die in $>$ Tab. 4 gelisteten Einflussfaktoren im Modell. Mit Ausnahme der Altersgruppe der Männer von 60 bis 69 Jahre ergab sich für alle Merkmale ein statistisch signifikanter Zusammenhang zur abhängigen Variable „mindestens ein neu aufgetretener Dekubitus im Pflegeheim “ (ja/nein) ( $\vdash$ Tab. 4). Das Vorliegen der Einflussfaktoren (außer EA) erhöht die Wahrscheinlichkeit für das Neuauftreten von Dekubitus im Jahresverlauf. Am stärksten ließ sich dies für die Pflegestufe III (inklusive der Härtefälle) feststellen: Hier war im Falle einer Schwerstpflegebedürftigkeit des Bewohners das Risiko für dieses Ereignis mehr als vervierfacht im Vergleich zu einem Bewohner mit Pflegestufe 1 (OR =4,61 (4,36-4,88 $\mathrm{Cl}, 95 \%)$ ), bei Vorliegen einer $\mathrm{EA}$ um etwa $30 \%$ reduziert $(\mathrm{OR}=0,68$ $(0,65-0,71 \mathrm{Cl}, 95 \%))$.

Knapp die Hälfte aller Einrichtungen (46,0\%) wies mehr Bewohner mit neu aufgetretenem Dekubitus auf als angesichts der Risikostruktur zu erwarten wäre (SMR > 1), 7,2\% der Pflegeheime mindestens doppelt so viele Bewohner. Das pflegeheimübergreifende Spektrum an Indikatorwerten bleibt auch nach Berücksichtigung der dekubitusspezifischen Risikostruktur breit (SMR - 1. Quartil: <0,6; 2. Q.: 0,6-0,8; 3. Q.: 0,9-1,3; 4. Q.: 1,4-4,5). Einige Einrichtungen veränderten jedoch ihre Position auf der $x$-Achse. Für jede fünfte Einrichtung ergab sich ein Quartilshift (20,2\% von 3459 Pflegeheimen), und davon mehrheitlich um genau ein Quartil ( + 1 Quartil: 51,0\%; - 1 Quartil: 48,1\%; + /-2 Quartile: 0,9\%).

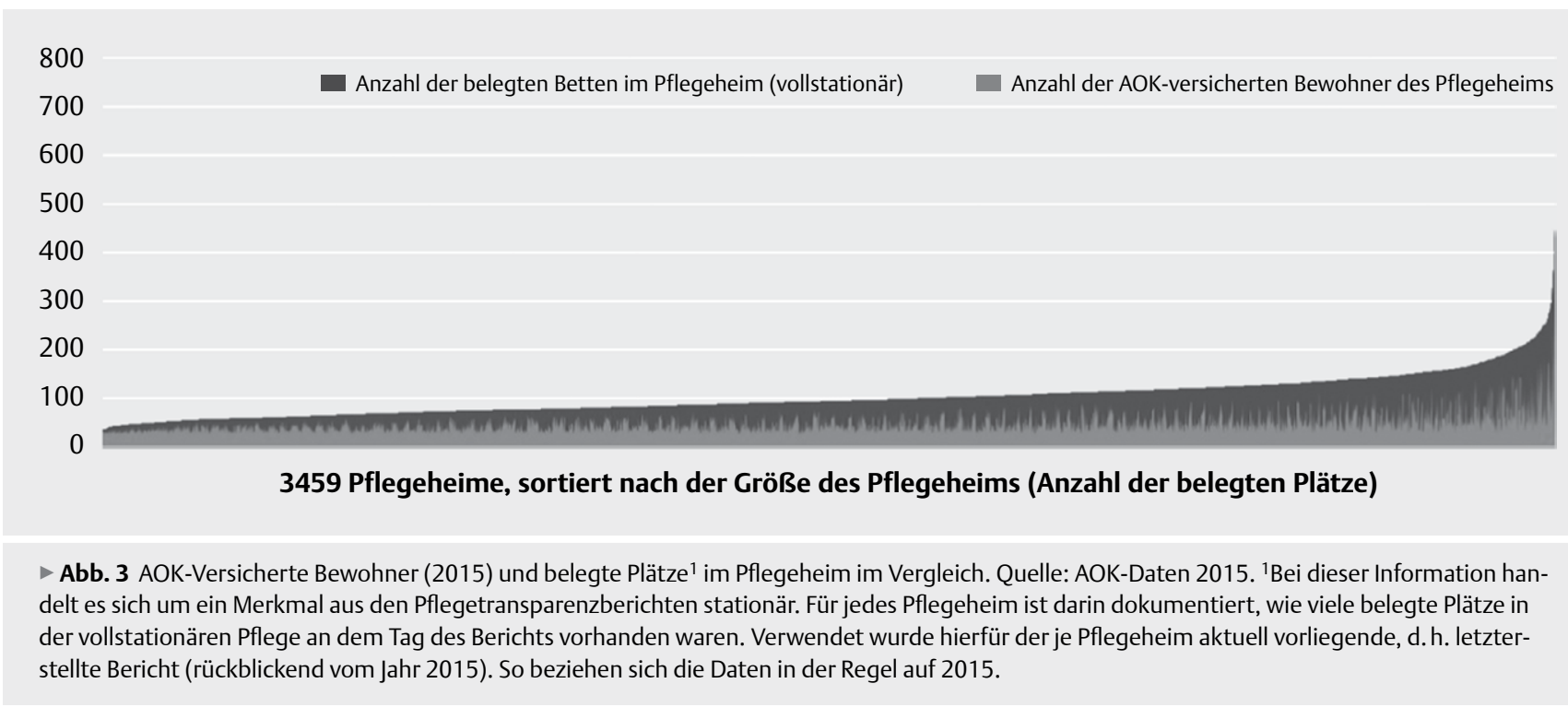




\section{Diskussion}

Die routinedatenbasierte Messung des Dekubitus-Auftretens im Pflegeheim ist, das zeigt die vorliegende Arbeit, machbar. Der Anteil der Bewohner mit mindestens einem in der Einrichtung neu aufgetretenen Dekubitus variiert erheblich zwischen den Einrichtungen. Die hier ermittelte routinedatenbasierte Versorgungsprävalenz von Dekubitus war konform mit jener eines aktuellen systematischen Reviews zur Epidemiologie des Dekubitus in Deutschland und speziell auch in der stationären Langzeitpflege [17]. Ebenso trifft dieses Ergebnis die Häufigkeit des aktuellen MDS-Berichts (4,0\% im Jahr 2015 vs. 3,9\% im Jahr 2016; [18]). Unter anderen aufgrund der geringen Stichprobengröße im Rahmen der MDK-Einrichtungsprüfung sind diese Dekubitus-Häufigkeiten im MDS-Bericht jedoch nicht als Validator der vorliegenden Daten zu verwenden. Auch eine Forschungsgruppe der Charité Berlin berechnete in der Langzeitstudie „Pflegeprobleme in Deutschland “ für 2011 eine ähnliche Prävalenz von 3,8\% (roh; Grad 1-4) in 50 deutschen Pflegeheimen (3 759 Bewohner) [34]. Die Dekubitus-Raten der Einrichtungen reichten in selbiger Untersuchung von $0 \%$ bis $13,6 \%$ der Pflegeheimbewohner. Gleichwohl ist zu beachten, dass die externe Validierung der Dekubitus-Inzidenz im Pflegeheim durch einen fehlenden Goldstandard erschwert ist. Das Spektrum an Inzidenzraten variiert primär in Abhängigkeit von den betrachteten Schweregraden, der Art und Anzahl der Einrichtungen und des Erhebungsverfahrens.

Die Vorteile ebenso wie die Limitationen von Routinedaten des Gesundheitswesens für Fragen der Versorgungsforschung, insbesondere im GKV-Kontext, sind hinlänglich beschrieben [20, 35]. So ist eine Einteilung in Schweregrade/Kategorien auf Basis vertrags- ärztlicher Abrechnungsdiagnosen (vierstelliger ICD-10-Code) auch hier nicht realisierbar - fast die Hälfte (46,2\%) der Dekubitus-Diagnosen waren unspezifisch kodiert (L89.9). Diese fehlende Differenzierung ist nicht unproblematisch: Dekubitus Schweregrad 1 beschreibt die nicht wegdrückbare Rötung und ist damit recht unspezifisch. Dementsprechend verzichten viele Studien auf diesen Schweregrad bzw. weisen Raten für Schweregrad 1 bis 4 und für Schweregrad 2 bis 4 aus [17, 34, 36, 37]. Der Aufgriff von Dekubitus mittels Diagnose plus ärztlicher Verordnung hydroaktiver Verbandsmaterialien in der vorliegenden Arbeit mindert diese Einschränkung jedoch, da Wundverbände in der Regel erst ab Schweregrad 2 erforderlich sind [13, 14]. Allerdings gilt zu beachten: Dass in Pflegeheimen Dekubiti, aufgegriffen durch Diagnose und Verbandsverordnung, messbar sind, ließe sich auch als (positiver) Hinweis werten, dass eine Versorgung stattfindet. Demgegenüber treten in Einrichtungen ohne entsprechende Dekubitus-Behandlung (d. h. ohne Verordnungen von Verbandsmaterial bei dokumentierter Diagnose Dekubitus) gemäß Indikatorfalldefinition scheinbar keine Fälle auf.

Eine weitere Limitation der Routinedatenanalyse bezieht sich auf das Inzidenzkriterium des Indikatorfalls: um ausschließlich die im Pflegeheim neu aufgetretenen Dekubiti zu erfassen, schloss die Falldefinition alle Bewohner mit einer Krankenhaus-Diagnose Dekubitus im Vorquartal aus. 14,5\% der Bewohner mit inzidentem Dekubitus wiesen jedoch eine derartige Krankenhausdiagnose im Quartal des Indikatorfalls auf. Hier ist aufgrund des Quartalsbezugs der Vertragsarztdaten die zeitliche (und damit ggf. ursächliche) Abfolge von Krankenhaus- und ambulant-ärztlicher Diagnose Dekubitus nicht zu bestimmen. Gleichwohl zeigt dieser Anteilswert, dass ein Ausschluss

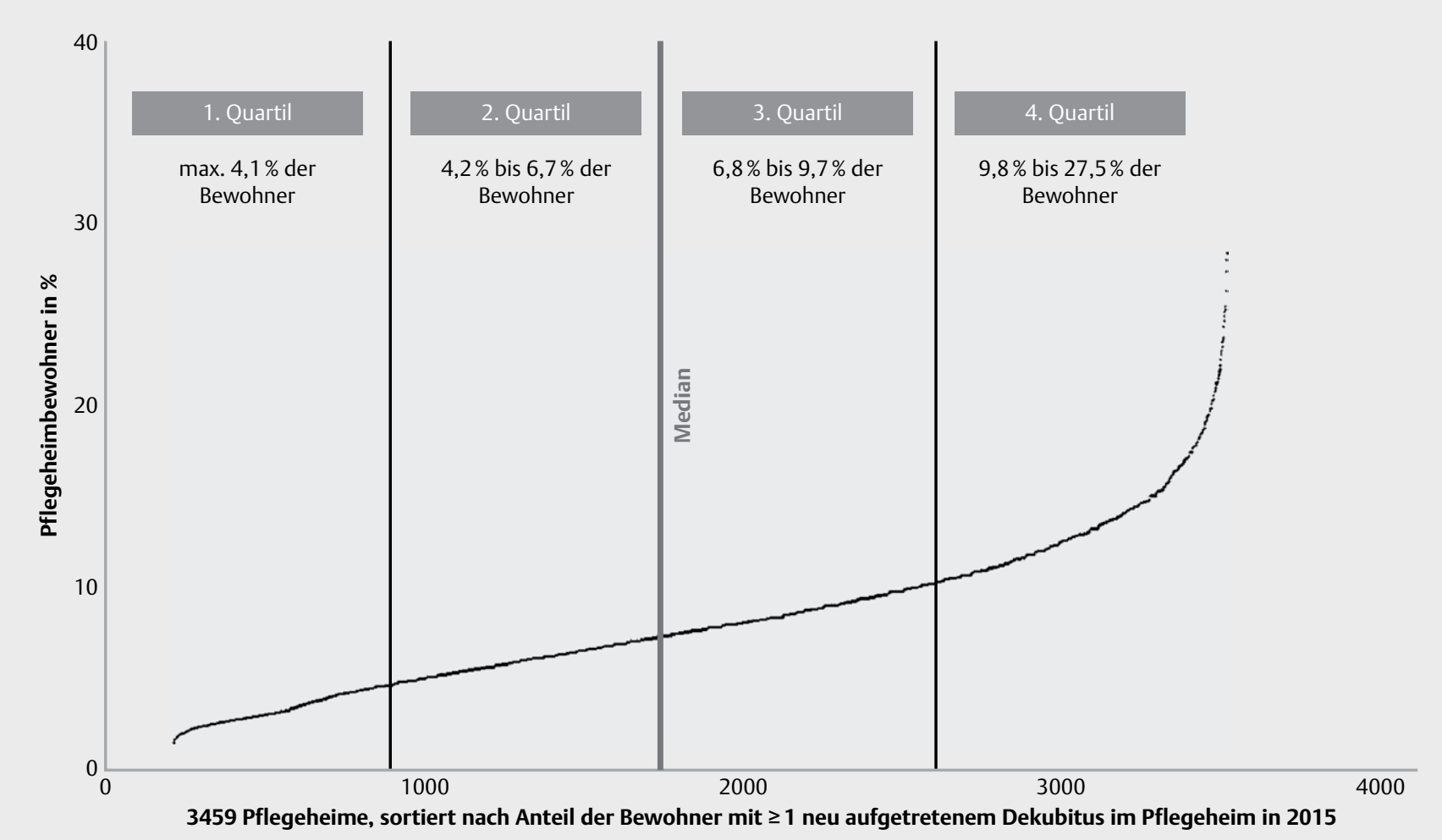

- Abb. 4 Bewohner mit mindestens einem neu aufgetretenen Dekubitus über die Pflegeheime hinweg. Quelle: AOK-Daten 2015. 
- Tab. 4 Risikoadjustierung Dekubitus: Ergebnisse der logistischen Regression. Quelle: AOK-Daten 2015.

\begin{tabular}{|c|c|c|c|c|}
\hline & \multirow[t]{2}{*}{ p-Wert } & \multicolumn{3}{|c|}{ Odds Ratio (OR, $95 \% \mathrm{Cl}$ ) } \\
\hline & & OR & $2,50 \%$ & $97,50 \%$ \\
\hline \multicolumn{5}{|l|}{ Frauen } \\
\hline \multicolumn{5}{|l|}{ 60-69 Jahre ${ }^{a}$} \\
\hline 70-79 Jahre & ${ }^{* *}$ & 1,15 & 1,00 & 1,31 \\
\hline 80-89 Jahre & *** & 1,25 & 1,11 & 1,42 \\
\hline $90+$ Jahre & $* * *$ & 1,43 & 1,26 & 1,63 \\
\hline \multicolumn{5}{|l|}{ Männer } \\
\hline 60-69 Jahre & n.s. & 1,14 & 0,97 & 1,33 \\
\hline 70-79 Jahre & $* * *$ & 1,31 & 1,14 & 1,50 \\
\hline 80-89 Jahre & $* * *$ & 1,35 & 1,18 & 1,54 \\
\hline $90+$ Jahre & $* * *$ & 1,55 & 1,34 & 1,80 \\
\hline \multicolumn{5}{|l|}{ Pflegestufe } \\
\hline \multicolumn{5}{|l|}{ Ia } \\
\hline II & $* * *$ & 2,69 & 2,56 & 2,84 \\
\hline III inklusive Härtefälle & $* * *$ & 4,61 & 4,36 & 4,88 \\
\hline Eingeschränkte Alltagskompetenz (primär Demenz) & $* * *$ & 0,68 & 0,65 & 0,71 \\
\hline Einschränkungen der Mobilität und Aktivität & $* * *$ & 1,35 & 1,30 & 1,40 \\
\hline Malnutrition & $* * *$ & 1,42 & 1,35 & 1,49 \\
\hline Dehydration & $* * *$ & 1,23 & 1,18 & 1,29 \\
\hline Parkinson/Sensorik & ${ }^{* * *}$ & 1,22 & 1,17 & 1,27 \\
\hline Diabetes mellitus & $* * *$ & 1,29 & 1,24 & 1,34 \\
\hline Inkontinenz/Feuchtigkeit & $* * *$ & 1,36 & 1,31 & 1,41 \\
\hline Intercept & & 0,02 & 0,02 & 0,02 \\
\hline
\end{tabular}

jener Bewohner mit einem Krankenhausaufenthalt im DekubitusQuartal ebenso zu einer Verzerrung, nämlich zum Ausschluss der gerade vulnerablen Personen, führen würde. Wie scharf die Abgrenzung zum Entstehungsort Krankenhaus erfolgen soll, bleibt letztlich Frage des konkreten Verwendungskontexts des Indikators.

Vor dem Hintergrund der - mit wenigen Ausnahmen - erklärten Vermeidbarkeit der Dekubiti (siehe hierzu u. a. [38-40], verweisen die Indikatorergebnisse auf einen erheblichen Optimierungsbedarf im Hinblick auf die Dekubitusprophylaxe. Die trotz Adjustierung bestehende Varianz der Indikatorwerte über die Pflegeheime hinweg ebenso wie das geringe Pseudo- $\mathrm{R}^{2}$ des Regressionsmodells von 0,1 legt nahe, dass andere (strukturelle und Performanz-) Parameter des Pflegeheimsettings das Dekubitus-Auftreten in der Langzeitpflege beeinflussen. Dass $80 \%$ der Pflegeheime trotz Berücksichtigung des Risikoprofils im Quartil des unadjustierten Indikatorwerts verbleiben, unterstützen diese Sicht. Die Studienlage u. a. zu kulturellen, organisatorischen und personalseitigen Bedingungsfaktoren für die Dekubitusgenese im Pflegeheim ist dicht (u. a. [40-43]. Zu prüfen ist zum anderen, inwiefern das Modell um weitere bewohnerbezogene Merkmale zu ergänzen ist. Castle et al. (2010) gibt zu bedenken, dass Faktoren wie Malnutration und Dehydration, vorliegend Bewohnercharakteristikum, durchaus auch als Prozessparameter zu verstehen sind [44]. Darüber hinaus gibt es unbekannte Risikofaktoren des Dekubitus (siehe $[13,41])$, relevante, jedoch nicht routinedatenbasiert abbildbare
Faktoren wie Vitalparameter [45] und schließlich nicht adjustierungsrelevante, von den Akteuren der Versorgung beeinflussbare, Faktoren [46-49]. Die Adjustierungsmethodik ist eines der Kernthemen im Rahmen der routinedatenbasierten Qualitätsmessung. Neben der Ausgestaltung des einzubeziehenden Risikofaktorensets auf inhaltlich-fachlicher Ebene ist es erforderlich, sich mit den multivariaten Verfahren der Risikoberücksichtigung auseinanderzusetzen und dabei neben Verfahren der logistischen und Poisson-Regression Nutzungskontext, Mehrwert und Limitationen von hierarchischen Modellen zu reflektieren und damit unter anderen nicht nur relevante Unterschiede der Bewohnerstruktur, sondern ebenso bspw. der Pflegeheimgrößen zu berücksichtigen.

Die Erfassung der Ergebnisqualität im Pflegeheim unter Rückgriff auf versichertenbezogene Abrechnungsdaten der Krankenund Pflegekassen ist demgegenüber vielversprechend: In Abgrenzung zu den zeitpunktbezogenen Qualitätsprüfungen durch die Prüfdienste der gesetzlichen Kranken- und Pflegekassen (Medizinische Dienste der Krankenversicherung, MDK) bzw. der Privaten Krankenversicherung (PKV) sowie zu den Eigendokumentationen der Pflegeheime (künftig einmal bzw. 2-mal jährlich; [1]) ermöglichen Routinedaten der GKV den Aufgriff aller sukzessiv im Jahr abgerechneten Fälle und damit eine deutliche Fallzahlerhöhung je Qualitätsindikator. Da jedes vierte Pflegeheim in Deutschland weniger als 30 Bewohner betreut, hat die Fallzahl-Prävalenz-Problematik (kleine Einrichtungen und seltene Ereignisse) hier eine er- 
hebliche Bedeutung [3]. Vor dem Hintergrund der Entbürokratisierungsdebatte und des Personalmangels in der Pflege haben routinedatenbasierte Indikatoren zur Ergebnisqualität im Pflegeheim darüber hinaus den entscheidenden Vorteil, mit keinen zusätzlichen Dokumentationspflichten verbunden zu sein.

\section{FAZIT}

Die routinedatenbasierte, indikatorgestützte Messung der Ergebnisqualität in der stationären Langzeitpflege steht am Anfang. Der hier ausgearbeitete Indikator Dekubitus ist insofern Prototyp, die Ausformulierung weiterer pflege- und gesundheitsbezogener Indikatoren und die Prüfung weiterer pflegebezogener bzw. pflegerelevanter Datenquellen müssen folgen. Zur Validierung der Vertragsarztdiagnosen von Pflegebedürftigen ist der Einbezug verfügbarer Hilfsmittelinformationen dabei naheliegend, da diese u. a. im Kontext pflegerelevanter Einschränkungen (Inkontinenz, Mobilitätseinschränkungen) verordnet werden. Auf bestehende Problemlagen (keine individuelle Verordnung, Heterogenität aufgrund des kassenindividuellen Vertragsgeschehens) wurde im Beitrag hingewiesen. Ebenso denkbar ist die stärkere Nutzung von Informationen aus der Pflegebegutachtung gemäß §18 SGB XI, um die pflegerischen Bedarfslagen über den Pflegegrad hinaus zu erfassen. Das Neue Begutachtungsassessement (NBA) zur Feststellung der Pflegebedürftigkeit liefert mit den 6 Modulen ein detailliertes Bild über die Fähigkeiten im Bereich Mobilität, kognitive und kommunikative Fähigkeiten, Verhaltensweisen und psychische Problemlagen, Selbstversorgung, Gestaltung des Alltagslebens und sozialer Kontakte [50]. Ferner bieten die ab 2019 novellierten Verfahren und Instrumente der Qualitätsprüfungen durch den MDK bzw. den Prüfdienst der PKV neue Möglichkeiten der Validierung von Indikatorwerten. Das Dekubitus-Auftreten ist dabei einer von 10 Indikatoren der externen gesetzlichen Qualitätssicherung in der Pflege [1].

Routinedatenbasierte Ergebnisindikatoren in der Langzeitpflege - in Kombination mit den im Rahmen einer novellierten Qualitätsmessung und -darstellung in der Langzeitpflege erhobenen Daten - eröffnen ferner neue Perspektiven für die Versorgungsforschung. Eine Frage fokussiert den Zusammenhang zwischen Ergebnisqualität und Personalausstattung. Ebenso ließen sich mit Indikatoren einrichtungsintern oder -übergreifend Maßnahmen zur Qualitätsverbesserung im Pflegeheim evaluieren. Um dieses erhebliche Potenzial zu nutzen, sollte ergänzend die wissenschaftliche Nutzung der Daten der novellierten Qualitätssicherung in der Pflege ermöglicht werden. Das aktuelle Gutachten, konzeptionelle Basis der Neuerung, sieht dies für pflegeepidemiologische und Qualitätsfragen zumindest vor.

\section{Interessenkonfllikt}

Die Autorinnen/Autoren geben an, dass kein Interessenkonflikt besteht.
Literatur

[1] Wingenfeld K, Stegbauer C, Willms G et al. Entwicklung der Instrumente und Verfahren für Qualitätsprüfungen nach $\S \S 114$ ff. SGB XI und die Qualitätsdarstellung nach § 115 Abs. 1a SGB XI in der stationären Pflege - Abschlussbericht: Darstellung der Konzeptionen für das neue Prüfverfahren und die Qualitätsdarstellung. Im Internet: https://www.gs-qsa-pflege.de/wp-content/up-

loads/2018/10/20180903_Entwicklungsauftrag_stationa\%CC\%88r_ Abschlussbericht.pdf; Stand: 30. Juli 2019. 2018

[2] Broge B, Stegbauer C, Woitzik R et al. Anforderungen an einen sektorenübergreifenden Zugang zu Qualität in der stationären Altenpflege. In: Jacobs K, Kuhlmey A, Greß S, Klauber J, Schwinger A, Hrsg. Pflege-Report 2018 - Schwerpunkt: Qualität in der Pflege. Berlin: Springer; 2018

[3] Schwinger A, Behrendt S. Reform der Qualitätsprüfung und -darstellung im Pflegeheim: Stand der Umsetzung und offene Fragen. GGW 2018; 18: 23-30

[4] SVR-Gesundheit Wettbewerb an der Schnittstelle zwischen ambulanter und stationärer Gesundheitsversorgung. Sondergutachten. Im Internet: http://dip21.bundestag.de/dip21/ btd/17/103/1710323.pdf; Stand: 26.11.2018 2012;

[5] CMS (2018) Design for Nursing Home Compare Five-Star Quality Rating System. Technical Users' Guide. February. 2018; Im Internet: https://www. cms.gov/Medicare/Provider-Enrollment-and-Certification/CertificationandComplianc/downloads/usersguide.pdf; Stand: 06.03.2018

[6] Przylog A, Stroka MA, Engel S et al. Do nursing homes with higher quality ratings provide a better quality of care? : Empirical study based on administrative data. Z Gerontol Geriatr 2016; 49: 308-316

[7] Dormann F, Klauber J, Kuhlen R. Qualitätsmonitor 2018. Berlin: Medizinisch Wissenschaftliche Verlagsgesellschaft; 2018

[8] IQTIG (2018) Methodische Grundlagen. Version V1.1s. Entwurf für das Stellungsnahmeverfahren. Stand: 15.11.2018. Im Internet: https:// iqtig.org/dateien/dasiqtig/grundlagen/IQTIG_Methodische-Grundlagen-V1.1s-2018-11-15.pdf; Stand: 26.11.2018

[9] Associates A (2018) Nursing Home Compare claims-based quality measures. Technical specifications. Final. Im Internet: https://www. cms.gov/Medicare/Provider-Enrollment-and-Certification/CertificationandComplianc/Downloads/Nursing-Home-Compare-Claims-basedMeasures-Technical-Specifications.pdf; Stand: 26.11.2018

[10] HQO (2017) Potentially avoidable emergency department visits for long-term care residents. Im Internet: http://indicatorlibrary. hqontario.ca/Indicator/Summary/Potentially-avoidable-2emergencydepartment-visits-LTC/EN

[11] Szecsenyi J, Stock J, Chenot R et al. QISA - das Qualitätsindikatorenset für die ambulante Vergütung. G\&S Gesundheits- und Sozialpolitik 2010; 64: 52-58

[12] GBE-Bund Dekubitus. Berlin: RKI; 2002

[13] DNQP (2017) Expertenstandard Dekubitusprophylaxe in der Pflege. Im Internet: https://www.dnqp.de/fileadmin/HSOS/Homepages/ DNQP/Dateien/Expertenstandards/Dekubitusprophylaxe_in_der_Pflege/Dekubitus_2Akt_Auszug.pdf; Stand: 26.06.2018

[14] NPUAP, EPUAP, PPPIA Prevention and Treatment of Pressure Ulcers: Quick Reference Guide. 2. Perth/Australia: Cambridge Media on behalf of National Pressure Ulcer Advisory Panel, European Pressure Ulcer Advisory Panel and Pan Pacific Pressure Injury Alliance; 2014

[15] Black JM, Edsberg LE, Baharestani MM et al. Pressure ulcers: avoidable or unavoidable? Results of the National Pressure Ulcer Advisory Panel Consensus Conference. Ostomy/wound management 2011; 57: 24-37

[16] Langemo DK, Black J. NPUAP Pressure Ulcers in Individuals Receiving Palliative Care: A National Pressure Ulcer Advisory Panel White Paper. Advances in skin \& wound care 2010; 23: 59-72

[17] Tomova-Simitchieva T, Akdeniz M, Blume-Peytavi U et al. The Epidemiology of Pressure Ulcer in Germany: Systematic Review. Gesundheitswesen 2018; 505-512 
[18] MDS (2018) Qualität in der ambulanten und stationären Pflege. 5. Pflege-Qualitätsbericht des MDS nach §114a Abs. 6 SGB XI. Im Internet: https://www.mds-ev.de/richtlinien-publikationen/pflegeversicherung/mds-pflege-qualitaetsberichte.html; Stand: 05.03.2018

[19] InBA (2017) Analysen der Diagnoseeinträge in der Datengrundlage des Bewertungsausschusses für die Ermittlung der diagnosebezogenen Veränderungsraten für das Jahr 2016. Im Internet: https://institut-ba. de/service/berichte.html; Stand: 28.11.2018

[20] Swart E, Ihle P, Gothe $\mathrm{H}$ et al. Routinedaten im Gesundheitswesen. Handbuch Sekundärdatenanalyse: Grundlagen, Methoden und Perspektiven. 2., vollständig überarbeitete und erweiterte Auflage. Bern: Verlag Hans Huber; 2014

[21] IGES (2012) Bewertung der Kodierqualität von vertragsärztlichen Diagnosen - Eine Studie im Auftrag des GKV-Spitzenverbands in Kooperation mit der BARMER GEK. Im Internet: https://www. gkv-spitzenverband.de/media/dokumente/krankenversicherung_1/ aerztliche_versorgung/verguetung_und_leistungen/klassifikationsver fahren/9_Endbericht_Kodierqualitaet_Hauptstudie_2012_12-19.pdf; Stand: 02.03.2018

[22] GKV-SV (2018) Hilfsmittelverzeichnis. Im Internet: https://hilfsmittel. gkv-spitzenverband.de/hmvAnzeigen.action?gruppeld =11\#orteTable; Stand: 26.11.2018

[23] Stemmer R, Arnold J. Expertise zur “Eignung von Indikatoren zur Messung und Darstellung von Ergebnisqualität in der stationären Pflege im Bereich der sozialen Pflegeversicherung". Im Internet: https://www.ikj-mainz.de/tl_files/Downloads/Pulikationen/Expertise \%20Ergebnisqualitaetsindikatoren \%20stationaere\%20Pflege_Stemmer\%20\&\%20Arnold_2014.pdf; Stand: 02.01.2018. 2014

[24] Becker A, Stausberg ], Fischer B et al. Risikoadjustierung von Qualitätsindikatoren. Eine Positionsbestimmung der DKG-Expertengruppe "Qualitätsmessung und Risikoadjustierung”. Das Krankenhaus 2016; 954-963

[25] Bottle A, Aylin P. Statistical Methods for Healthcare Performance Monitoring. 1 edition (Aug. 1) 2016Boca Raton, FL: CRC Press; 2016

[26] UBC (2017) Abschlussbericht zum Forschungsprojekt Modellhafte Pilotierung von Indikatoren in der stationären Pflege (MoPIP). Im Internet: https://www.gkv-spitzenverband.de/mwg-internal/ de5fs23hu73g7/progress?id = TK42E98TvcEdV7cFjeP9Ef9UBrm5M9EWkaQRra760Q,\&dl; Stand: 02.01.2018

[27] Berlowitz DR, Intrator O. Risk adjustment for Long-Term Care.In lezzoni IL, Hrsg. Risk Adjustment for Measuring Healthcare Outcomes, Fourth Edition . Fourth editionChicago: IL Health Administration Press; 2012: 423-442

[28] HQO (2015) LTC Indicator Review Report: The review and selection of indicators for long-term care public reporting. Im Internet: https:// www.hqontario.ca/Portals/0/documents/system-performance/ltc-indicator-review-report-november-2015.pdf; Stand: 12.02.2019

[29] Ash AS, Schwartz M, Peköz EA. Comparing outcomes across providers. In: lezzoni IL, Hrsg. Risk adjustment. $3^{\text {rd }}$ edition. Chicago, Illinois: Health Administration Press; 2003

[30] IQTIG (2017) Beschreibung der Qualitätsindikatoren für das Erfassungsjahr 2016. Pflege: Dekubitusprophylaxe. Im Internet: https:// iqtig.org/downloads/auswertung/2016/dek/QSKH_DEK_2016_QIDB_ V02_2017-04-26.pdf; Stand: 26.06.2018

[31] Meinck M, Lübke N. Geriatrietypische Multimorbidität im Spiegel von Routinedaten. Teil 3: Prävalenz und prädiktiver Wert geriatrietypischer Merkmalskomplexe in einer systematischen Altersstichprobe. Z Gerontol Geriat 2013; 645-657

[32] Petrie A, Sabin C. Medical Statistics at a Glance. Oxford: Wiley Blackwell; 2013

[33] Statistisches Bundesamt (2017) Pflegestatistik 2015 - Pflege im Rahmen der Pflegeversicherung - Deutschlandergebnisse. Im Internet: https://www.destatis.de/DE/Publikationen/Thematisch/Gesundheit/ Pflege/PflegeDeutschlandergebnisse5224001159004.pdf?_ blob = publicationFile; Stand: 02.03.2018
[34] Klingelhofer-Noe J, Dassen T, Lahmann NA. Nursing homes versus assisted living facilities: Outcome quality regarding pressure ulcers, falls and malnutrition. Z Gerontol Geriatr 2015; 48: 263-269

[35] Schubert I, Ihle P, Köster I et al. Daten für die Versorgungsforschung. Zugang und Nutzungsmöglichkeiten. Gutachten für das Deutsche Institut für Medizinische Dokumentation und Information (DIMDI). Im Internet: https://www.dimdi.de/static/.downloads/deutsch/ dimdi-sekundaerdaten-expertise.pdf; Stand: 26.11.2018. 2014

[36] IQTIG (2017) Bundesauswertung zum Erfassungsjahr 2016. Pflege: Dekubitusprophylaxe. Qualitätsindikatoren. Im Internet: https://iqtig. org/downloads/auswertung/2016/dek/QSKH_DEK_2016_BUAW_ V02_2017-07-12.pdf; Stand: 26.06.2018

[37] Kottner ], Dassen T, Lahmann NA. Pressure ulcers in German nursing homes: frequencies, grades, and origins. Z Gerontol Geriatr 2011; 44: 318-322

[38] Duncan KD. Preventing pressure ulcers: the goal is zero. Joint Commission Journal on Quality and Patient Safety 2007; 33: 605-611

[39] Black JM et al. Pressure ulcers: avoidable or unavoidable? Results of the National Pressure Ulcer Advisory Panel Consensus Conference. Ostomy Wound Management 2011; 57: 24-37

[40] Kottner ], Hahnel E, Lichterfeld-Kottner A et al. Measuring the quality of pressure ulcer prevention: A systematic mapping review of quality indicators. International wound journal 2018; 15: 218-224

[41] Lavallée JF, Gray TA, Dumville J et al. Barriers and facilitators to preventing pressure ulcers in nursing home residents: A qualitative analysis informed by the Theoretical Domains Framework. International Journal of Nursing Studies 2018; 82: 79-89

[42] Sullivan N, Schoelles KM. Preventing in-facility pressure ulcers as a patient safety strategy. A systematic review. Annals of Internal Medicine 2013; 158: 410-416

[43] Krause T, Anders ], Von Renteln-Kruse W. Pressure sores: knowledge of risk factors and awareness of problems with quality of care as reflected by questionnaire answers by nursing staff and physicians. Z Gerontol Geriatr 2004; 37: 86-91

[44] Castle NG, Ferguson JC. What is nursing home quality and how is it measured? Gerontologist 2010; 50: 426-442

[45] PMVforschungsgruppe Gutachten: Daten für die Versorgungsforschung. Zugang und Nutzungsmöglichkeiten. Im Internet: https:// www.dimdi.de/static/.downloads/deutsch/dimdi-sekundaerdatenexpertise.pdf; Stand: 12.07.2019. 2014

[46] Spector WD, Mukamel DB. Using Outcomes to Make Inferences about Nursing Home Quality. Evaluation \& the Health Professions 1998; 21: 291-315

[47] Mant. Process versus outcome indicators in the assessment of quality of health care. International Journal for Quality in Health Care 2001; 13: $475-480$

[48] Hasseler M, Wolf-Ostermann K. Wissenschaftliche Evaluation zur Beurteilung der Pflege-Transparenzvereinbarungen für den ambulanten (PTVA) und stationären (PTVS) Bereich. Im Internet: https://www. gkv-spitzenverband.de/mwg-internal/de5fs23hu73g7/ progress?id = jzURmZKOSsHTowXyln-2cAWxZxAmdb9gkPy6r3ZoWU,\&dl; Stand: 19.10.2016. 2010

[49] Rothgang H. Qualität in der Langzeitpflege. In: Welti F, Fuchs M, Fuchsloch C, Naegele G, Udsching P, Hrsg. Gesundheit, Alter, Pflege, Rehabilitation - Recht und Praxis im interdisziplinären Dialog. Festschrift für Gerhard I II.Nomos 2017; 630-646

[50] MDS (2017) Das Neue Begutachtungsinstrument der Sozialen Pflegeversicherung. Die Selbstständigkeit als Maß der Pflegebedürftigkeit. Im Internet: https://www.mds-ev.de/fileadmin/dokumente/ Publikationen/SPV/Begutachtungsgrundlagen/Fachinfo_PSGII_web_ neu_Feb_2017.pdf; Stand: 11.12.2018 